\title{
通过点击化学方法合成鹅去氧胆酸类分子钳及其识别性能研究
}

\author{
赵志刚*＼cjkstart王晓红＼cjkstart石治川＼cjkstart⿰⿱㇒木⿱口王玉宇 \\ (西南民族大学化学与环境保护工程学院＼cjkstart成都 610041)
}

\begin{abstract}
摘要 通过点击化学方法设计合成了 12 个新型的含有 $1,2,3$-三唑结构的鹅去氧胆酸类分子针人工受体. 所有新目标物 结构均经 ${ }^{1}$ H NMR, IR, MS 及元素分析确证. 利用紫外可见光光度滴定法测试了主体化合物对阳离子的识别作用. 实验 结果表明, 主体分子对录离子有良好的选择性识别性能.
\end{abstract}

关键词 阳离子识别; 分子钳; 点击化学; $1,2,3$-三唑; 我去氧胆酸

\section{Synthesis of Molecular Tweezers Derived from Chenodeoxycholic Acid through Click Reaction and Their Recogniton Properties}

\author{
Zhao, Zhigang* Wang, Xiaohong Shi, Zhichuan Cheng, Yuyu \\ (College of Chemistry and Environmental Protection Engineering, Southwest University for Nationalities, Chengdu 610041)
}

\begin{abstract}
Twelve novel chenodeoxycholic acid-based molecular tweezer metal receptors containing 1,2,3-triazole moieties have been designed and synthesized using click chemistry method. The target compounds were characterized by ${ }^{1} \mathrm{H}$ NMR, IR, MS spectra and elemental analysis. Their binding properties were examined by UV-Vis spectra titration. The results indicate that these molecular tweezers showed high selectivity and affinity for $\mathrm{Hg}^{2+}$ ion.

Keywords cation recognition; molecular tweezer; click chemistry; 1,2,3-triazole; chenodeoxycholic acid
\end{abstract}

金属阳离子广泛存在于生物体内和我们的生活环 境中. 在生物体代谢过程中, 金属阳离子起着特殊的作 用，它们维持着生物体内的水和电解质平衡以及参与 DNA 及酶的代谢过程, 是人体细胞不可或缺的 ${ }^{[1]}$. 在工 业生产中, 广泛使用铅、录、镉等剧毒重金属, 它们大 都转化成为阳离子被排放到自然界中, 通过食物链在组 织里富集起来, 并转化为人体细胞内游离的阳离子, 对 人和自然界造成巨大的危害 ${ }^{[2,3]}$. 设计易于合成且能够 准确、快速检测出有毒金属阳离子的人工受体是目前研 究阳离子识别的一个热点领域 ${ }^{[4]} .2001$ 年, Sharpless 等 ${ }^{[57]}$ 提出了一种快速合成大量化合物的方法, 即“click chemistry”，其具有反应条件温和、选择性高、产率高、 纯化步骤简单等优点. 通过点击化学方法合成含有 1,2,3-三曲结构的多性能的化合物成为近几年的一个研 究热点 ${ }^{[8 \sim 13]}$. 1,2,3-三唑结构具有较大的偶极矩, 可参与 形成氢键. 同时, 在比较苛刻的条件下，仍具有很高的 稳定性. 另外 $1,2,3-$ 三唑结构能够参与 $\pi-\pi$ stacking 和配
位作用，故可与客体分子之间发生良好的识别作用. 由 于 1,2,3-三唑结构具有如此多优点，故将其应用于合成 具有特殊识别功能的分子钳具有重要的理论意义与实 用价值. 甾体由于其具有刚性的凹面结构和天然的不对 称性, 是构筑钳形人工受体的理想结构单元 ${ }^{[14 ~ 20]}$. 通过 点击化学合成甾体类分子钳的研究, 国内外报道相对较

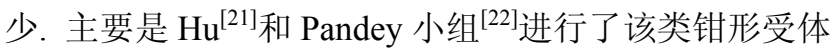
的合成及性能测试. 结果表明, 对一些金属阳离子有良 好的识别性能. 纵观国内外文献, 利用点击化学来合成 我去氧胆酸钳形人工受体还未见文献报道, 因此我们设 计了一类新型的鹅去氧胆酸分子钳. 以鹅去氧胆酸为隔 离基, 经过桥连得到 3,7-二叠氮乙酰氧基㧶去氧胆酸甲 酯, 通过点击化学方法合成目标分子钳人工受体. 紫外 可见光谱滴定结果表明, 这类钳形受体对阳离子具有识 别作用, 特别对录离子有优良的选择性识别效果. 合成 路线见 Scheme 1.

* E-mail: zzg63129@163.com

Received January 25, 2014; revised March 10, 2014; published online April 15, 2014.

Project supported by the Science and Technology Department of Sichuan Province (No. 2012SZ0160) and the National Foreign Expert Bureau (No. 2013-32).

四川省科技支撑计划基金(No. 2012SZ0160)和国家外专局基金(No. 2013-32)资助项目. 

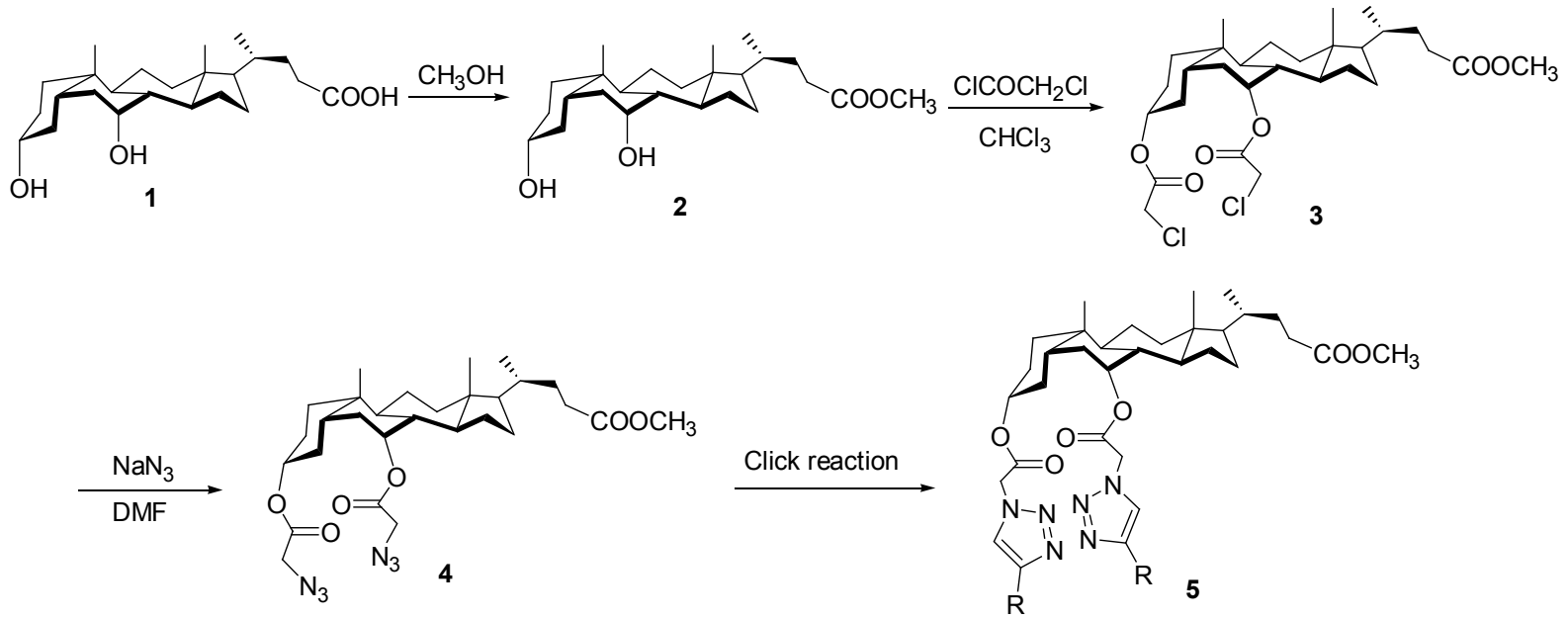<smiles>[R16]C#[R]Cc1cccc(C#[R]Cc2ccc(OC)cc2)c1</smiles>

5e: $R=$

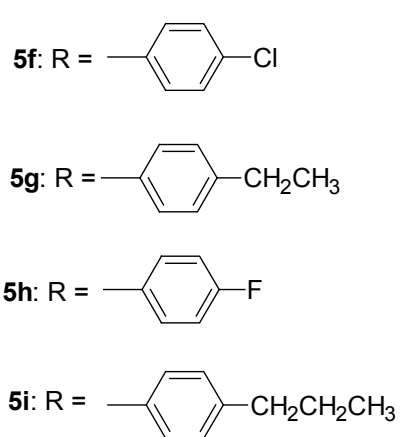

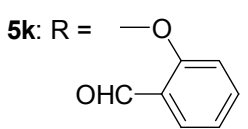

5I: R=

Scheme 1

\section{1 实验}

\section{1 仪器与试剂}

核磁共振仪: Varian INOVA400 $\mathrm{MHz}, \mathrm{Me}_{4} \mathrm{Si}$ 作内 标, $\mathrm{CDCl}_{3}, \mathrm{CDCl}_{3} \mathrm{CD}_{3} \mathrm{OD}$ 混合溶液为溶剂; 红外光谱仪: 傅里叶变换红外光谱仪 PERKINEL-MER1700 型, KBr 压片; 质谱仪: FINNIGAN-LCQ ${ }^{\text {DECA }}$ 型; 元素分析仪: Carlo-Erba-1106 型; 旋光仪: $\mathrm{W}_{\mathrm{ZZ}}-2 \mathrm{~B}$ 自动旋光仪; 熔点 仪: X-4 型数字显示显微熔点测定仪, 温度计未经校正; 紫外可见光谱仪: 北京普析 TU-1901 型.

所用试剂均为市售化学纯或分析纯. 硅胶 $\mathrm{H}$ 、硅胶 $\mathrm{GF}_{254}$ : 青岛海洋化工厂生产; 无水 $N, N$-二甲基甲酰胺 (DMF): 加 $\mathrm{CaH}_{2}$ 干燥 $48 \mathrm{~h}$ 后, 反应前新蒸使用; 无水吡 啶: 加 $\mathrm{KOH}$ 干燥 $24 \mathrm{~h}$ 后, 蒸馏收集 114 $116{ }^{\circ} \mathrm{C}$ 馏分; 鹅去氧胆酸: Acros Organics 公司进口; 对硝基苯乙炔: Sigma 公司生产; 对甲基苯乙炔: Aladdin 公司生产; 对 丙基苯乙炔: Adamas-beta Reagent 公司生产; 2-(2-丙炔 氧基)苯甲醛、2-(2-丙炔氧基)-1-菜甲醛：J\&K Chemical 公司进口; 苯乙炔、4-乙基苯乙炔、4-甲氧基苯乙炔、 对溴苯乙炔、对氯苯乙炔、对氟苯乙炔、3-氨基苯乙炔: 上海徳默医药科技有限公司生产.

\section{2 中间体 2 4 的合成与表征}

$3 \alpha, 7 \alpha$-二羟基- $5 \beta-24$-胆烷酸甲酯(2)的合成: 将 $2.0 \mathrm{~g}$ (5.0 mmol)鹅去氧胆酸 $\mathbf{1}$ 加入 $100 \mathrm{~mL}$ 圆底烧瓶中，依次 加入 $60 \mathrm{~mL}$ 无水甲醇和 $0.2 \mathrm{~g}(1.16 \mathrm{mmol})$ 对甲苯磺酸, 室温下搅拌反应 $24 \mathrm{~h}$. 减压蒸去甲醇, 剩余物用 $40 \mathrm{~mL}$ 乙酸乙酯溶解，然后用 $10 \%$ 碳酸钠溶液 $(10 \mathrm{~mL} \times 3)$ 洗涤, 再用饱和食盐水 $(10 \mathrm{~mL} \times 3)$ 洗涤, 无水硫酸钠干燥, 过 滤. 减压蒸去乙酸乙酯后, 柱层析分离纯化[固定相用 硅胶 $\mathrm{H}$, 洗脱剂: $V$ (甲醇) $: V$ (丙酮 $): V($ 氯仿 $)=2: 20$ : 70 ], 得白色固体 $1.92 \mathrm{~g}$, 产率 $95 \%$. m.p. $85 \sim 86{ }^{\circ} \mathrm{C}$; $\left.[\alpha]_{\mathrm{D}}^{20}+18.9(c) 0.34, \mathrm{CH}_{2} \mathrm{Cl}_{2}\right) ;{ }^{1} \mathrm{H}$ NMR $(400 \mathrm{MHz}$, $\left.\mathrm{CDCl}_{3}\right) \delta: 3.85(\mathrm{~s}, 1 \mathrm{H}, 7 \beta-\mathrm{H}), 3.66\left(\mathrm{~s}, 3 \mathrm{H}, \mathrm{COOCH}_{3}\right)$, $3.50 \sim 3.42(\mathrm{~m}, 1 \mathrm{H}, 3 \beta-\mathrm{H}), 0.93\left(\mathrm{~s}, 3 \mathrm{H}, 19-\mathrm{CH}_{3}\right), 0.91(\mathrm{~d}$, $\left.J=6.4 \mathrm{~Hz}, 3 \mathrm{H}, 21-\mathrm{CH}_{3}\right), 0.66$ (s, 3H, 18- $\left.\mathrm{CH}_{3}\right)$; IR (KBr) $v$ : 3350, 2985, 2910, 1745, $1183 \mathrm{~cm}^{-1}$; ESI-MS $m / z$ (\%): 405 $\left[(\mathrm{M}-\mathrm{H})^{+}, 100\right]$.

$3 \alpha, 7 \alpha$-二氯乙酰氧基- $5 \beta$ - 24 -胆烷酸甲酯 (3) 的合成: 将 $0.81 \mathrm{~g}(2.0 \mathrm{mml}) 3 \alpha, \alpha$-二羟基- $5 \beta$-24-胆烷酸甲酯(2)溶 于 $40 \mathrm{~mL}$ 无水 $\mathrm{CHCl}_{3}$ 中, 再加入 $0.64 \mathrm{~mL}(8.0 \mathrm{mml})$ 的 $\mathrm{ClCH}_{2} \mathrm{COCl}$, 室温搅拌下滴加吡啶的 $\mathrm{CHCl}_{3}$ 溶液 $(0.30$ $\mathrm{mL} / 3.70 \mathrm{mmol}$ 溶于 $10 \mathrm{~mL}$ 无水 $\left.\mathrm{CHCl}_{3}\right)$, 滴加完毕后, 升 温至 $60{ }^{\circ} \mathrm{C}$ 回流 $48 \mathrm{~h}$. 停止反应后, 减压蒸出多余的 
$\mathrm{CHCl}_{3}$ 和过量 $\mathrm{ClCH}_{2} \mathrm{COCl}$, 得乳黄色固体, 残余物用乙 酸乙酯溶解, 依次用饱和碳酸氢钠 $(30 \mathrm{~mL} \times 3)$, 饱和食 盐水 $(30 \mathrm{~mL} \times 3)$ 洗涤, 无水硫酸钠干燥. 经硅胶柱层析 [固定相用硅胶 $\mathrm{H}$, 洗脱剂: $V$ (乙酸乙酯) $: V$ (石油醚 $)=$ $1: 5$ ]得白色固体 $1.00 \mathrm{~g}$, 产率 $90 \%$. m.p. $120 \sim 122{ }^{\circ} \mathrm{C}$; $[\alpha]_{\mathrm{D}}^{20}-71.8\left(c \quad 0.19, \mathrm{CH}_{2} \mathrm{Cl}_{2}\right) ;{ }^{1} \mathrm{H}$ NMR $\left(\mathrm{CDCl}_{3}, 400\right.$ MHz) $\delta: 5.00(\mathrm{~s}, 1 \mathrm{H}, 7 \beta-\mathrm{H}), 4.66 \sim 4.70(\mathrm{~m}, 1 \mathrm{H}, 3 \beta-\mathrm{H})$, $3.99 \sim 4.08\left(\mathrm{~m}, 4 \mathrm{H}, \mathrm{CH}_{2} \mathrm{Cl}\right), 3.67$ (s, $\left.3 \mathrm{H}, \mathrm{COOCH}_{3}\right), 0.95$ (s, 3H, 19- $\mathrm{CH}_{3}$ ), 0.92 (d, $\left.J=6.4 \mathrm{~Hz}, 3 \mathrm{H}, 21-\mathrm{CH}_{3}\right), 0.65$ (s, $\left.3 \mathrm{H}, 18-\mathrm{CH}_{3}\right)$; IR (KBr) v: 2946, 2872, 1752, 1735, 1438, 1420, 1304, 1247, 1191, 1134, 1059, 985, $970 \mathrm{~cm}^{-1}$; ESI-MS $m / z(\%): 581\left[(\mathrm{M}+\mathrm{Na})^{+}, 100\right]$. Anal. calcd for $\mathrm{C}_{29} \mathrm{H}_{45} \mathrm{C}_{12} \mathrm{O}_{6}$ : C 62.25, H 7.93; found C 62.18, H 7.95.

$3 \alpha, 7 \alpha$-二叠氮乙酰氧基- $5 \beta$ - 24 -胆烷酸甲酯(4)的合 成: 将 $0.86 \mathrm{~g}$ (1.54 mmol) $3 \alpha, 7 \alpha$-二氯乙酰氧基- $5 \beta$-24-胆

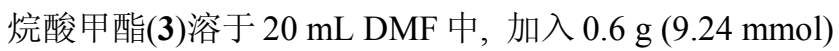
叠氮化钠. $60{ }^{\circ} \mathrm{C}$ 条件下反应 $12 \mathrm{~h}$. 停止反应之后, 加入 $40 \mathrm{~mL}$ 水稀释反应液, 用乙酸乙酯 $(30 \mathrm{~mL} \times 3)$ 萃取, 合 并有机相, 依次用水和饱和氯化钠溶液各洗涤 1 次, 无 水硫酸钠干燥. 柱层析后[固定相用硅胶 $\mathrm{H}$, 洗脱剂为: $V$ (乙酸乙酯)： $V($ 石油醚 $)=1: 5$ ] 得白色固体 $0.84 \mathrm{~g}$, 产 率 95\%. m.p. 81 $83{ }^{\circ} \mathrm{C} ;[\alpha]_{\mathrm{D}}^{20}-108.3\left(c 0.11, \mathrm{CH}_{2} \mathrm{Cl}_{2}\right)$; ${ }^{1} \mathrm{H}$ NMR $\left(\mathrm{CDCl}_{3}, 400 \mathrm{MHz}\right) \delta: 5.04(\mathrm{~s}, 1 \mathrm{H}, 7 \beta-\mathrm{H}), 4.70 \sim$ $4.75(\mathrm{~m}, 1 \mathrm{H}, 3 \beta-\mathrm{H}), 3.79 \sim 3.92\left(\mathrm{~m}, 4 \mathrm{H}, \mathrm{CH}_{2} \mathrm{~N}_{3}\right), 3.66(\mathrm{~s}$, $\left.3 \mathrm{H}, \mathrm{COOCH}_{3}\right), 0.96\left(\mathrm{~s}, 3 \mathrm{H}, 19-\mathrm{CH}_{3}\right), 0.92(\mathrm{~d}, J=6.4 \mathrm{~Hz}$, $\left.3 \mathrm{H}, 21-\mathrm{CH}_{3}\right), 0.66\left(\mathrm{~s}, 3 \mathrm{H}, 18-\mathrm{CH}_{3}\right)$; IR ( $\left.\mathrm{KBr}\right) v: 2945$, 2867, 2110, 1742, 1376, 1349, 1291, 1249, 1200, 1165, 1140, $984 \mathrm{~cm}^{-1}$; ESI-MS $\mathrm{m} / z(\%): 595\left[(\mathrm{M}+\mathrm{Na})^{+}, 100\right]$. Anal. calcd for $\mathrm{C}_{29} \mathrm{H}_{45} \mathrm{~N}_{6} \mathrm{O}_{6}$ : C 60.82, H 7.74, N 14.67; found $\mathrm{C} 60.74, \mathrm{H} 7.75, \mathrm{~N} 14.65$.

\section{3 分子钳 $5 a \sim 5$ 的合成与表征}

在 $50 \mathrm{~mL}$ 干燥的圆底烧瓶中加入 $0.3 \mathrm{~g}(0.52 \mathrm{mmol})$ $3 \alpha, 7 \alpha$-二叠氮乙酰氧基- $5 \beta-24$-胆烷酸甲酯 (4), $15 \mathrm{~mL}$ $t-\mathrm{BuOH}$, 待原料溶解完后, 加入各种取代苯乙炔 1.14 $\mathrm{mmol}, 40 \mathrm{~mol} \%$ 三乙胺, $10 \mathrm{~mol} \% \mathrm{CuI}$, 油浴加热, 在 50 ${ }^{\circ} \mathrm{C}$ 反应 10 30 h ( TLC 监测反应进程), 反应停止后, 减 压蒸出多余的 $t-\mathrm{BuOH}$, 得乳白色固体, 残余物用少量 乙酸乙酯溶解. 经硅胶柱层析[固定相用硅胶 $\mathrm{H}$, 洗脱 剂: $V($ 乙酸乙酯 $): V($ 石油醚 $)=3: 10 \sim 2: 1$ ]得到目标 物.

分子钳 5a: 白色固体，产率 93\%. m.p. 97 99 ${ }^{\circ} \mathrm{C}$; $[\alpha]_{D}^{20}+242.3\left(c \quad 0.20, \mathrm{CH}_{2} \mathrm{Cl}_{2}\right) ;{ }^{1} \mathrm{H}$ NMR $(400 \mathrm{MHz}$, $\left.\mathrm{CDCl}_{3}\right) \delta: 8.19(\mathrm{~s}, 1 \mathrm{H}$, triazole- $\mathrm{H}), 7.86(\mathrm{~s}, 1 \mathrm{H}$, triazole- $\mathrm{H})$, $7.81 \sim 7.84(\mathrm{~m}, 4 \mathrm{H}, \operatorname{ArH}), 7.42 \sim 7.46(\mathrm{~m}, 2 \mathrm{H}, \operatorname{ArH})$, $7.34 \sim 7.38(\mathrm{~m}, 3 \mathrm{H}, \operatorname{ArH}), 7.28 \sim 7.31(\mathrm{~m}, 1 \mathrm{H}, \operatorname{ArH})$,
$5.25 \sim 5.41\left(\mathrm{~m}, 2 \mathrm{H}, \mathrm{OCOCH}_{2}\right), 5.04 \sim 5.12(\mathrm{~m}, 2 \mathrm{H}$, $\left.\mathrm{OCOCH}_{2}\right), 4.92(\mathrm{~s}, 1 \mathrm{H}, 7 \beta-\mathrm{H}), 4.61 \sim 4.65(\mathrm{~m}, 1 \mathrm{H}, 3 \beta-\mathrm{H})$, 3.68 (s, 3H, $\left.\mathrm{COOCH}_{3}\right), 0.89$ (s, 3H, 19- $\left.\mathrm{CH}_{3}\right), 0.84$ (d, $J=$ $\left.6.4 \mathrm{~Hz}, 3 \mathrm{H}, 21-\mathrm{CH}_{3}\right), 0.59$ (s, 3H, 18- $\left.\mathrm{CH}_{3}\right)$; IR $(\mathrm{KBr}) v$ : 2943, 2869, 1738, 1466, 1440, 1364, 1276, 1221, 1046, 976, 765, $695 \mathrm{~cm}^{-1}$; ESI-MS $\mathrm{m} / z(\%): 777\left[(\mathrm{M}+\mathrm{H})^{+}\right.$, 100]. Anal. calcd for $\mathrm{C}_{45} \mathrm{H}_{56} \mathrm{~N}_{6} \mathrm{O}_{6}: \mathrm{C} 69.56, \mathrm{H}$ 7.26, N 10.82; found C 69.52, H 7.24, N 10.78.

分子钳 5b: 白色固体, 产率 $88 \%$. m.p. $96 \sim 98{ }^{\circ} \mathrm{C}$; $[\alpha]_{\mathrm{D}}^{20}-42.9$ (c $\left.0.18, \mathrm{CH}_{2} \mathrm{Cl}_{2}\right) ;{ }^{1} \mathrm{H}$ NMR $(400 \mathrm{MHz}$, $\left.\mathrm{CDCl}_{3}\right) \delta: 8.16(\mathrm{~s}, 1 \mathrm{H}$, triazole-H), $7.80(\mathrm{~s}, 1 \mathrm{H}$, triazole- $\mathrm{H})$, $7.72 \sim 7.76$ (m, 4H, ArH), 7.26 7.28 (m, 2H, ArH), 7.18 (d, $J=8 \mathrm{~Hz}, 2 \mathrm{H}, \mathrm{ArH}), 5.23 \sim 5.39\left(\mathrm{~m}, 2 \mathrm{H}, \mathrm{OCOCH}_{2}\right)$, $5.02 \sim 5.13\left(\mathrm{~m}, 2 \mathrm{H}, \mathrm{OCOCH}_{2}\right), 4.91(\mathrm{~s}, 1 \mathrm{H}, 7 \beta-\mathrm{H}), 4.60 \sim$ $4.66(\mathrm{~m}, 1 \mathrm{H}, 3 \beta-\mathrm{H}), 3.68\left(\mathrm{~s}, 3 \mathrm{H}, \mathrm{COOCH}_{3}\right), 2.60(\mathrm{brs}, 4 \mathrm{H}$, $\left.\mathrm{ArNH}_{2} \times 2\right), 0.88\left(\mathrm{~s}, 3 \mathrm{H}, 19-\mathrm{CH}_{3}\right), 0.82(\mathrm{~d}, J=6.4 \mathrm{~Hz}, 3 \mathrm{H}$, 21- $\mathrm{CH}_{3}$ ), 0.58 (s, 3H, 18- $\mathrm{CH}_{3}$ ); IR (KBr) v: 2942, 2871, 1741, 1507, 1459, 1363, 1275, 1227, 1139, 1045, 977, 831, $790 \mathrm{~cm}^{-1}$; ESI-MS $\mathrm{m} / z(\%): 807\left[(\mathrm{M}+\mathrm{H})^{+}, 100\right]$. Anal. calcd for $\mathrm{C}_{45} \mathrm{H}_{58} \mathrm{~N}_{8} \mathrm{O}_{6}$ : C 66.97, H 7.24, N 13.89; found C 66.86, H 7.23, N 13.92 .

分子钳 5c: 白色固体, 产率 $91 \%$. m.p. $98 \sim 100{ }^{\circ} \mathrm{C}$; $[\alpha]_{\mathrm{D}}^{20}-56.8\left(c \quad 0.16, \mathrm{CH}_{2} \mathrm{Cl}_{2}\right) ;{ }^{1} \mathrm{H}$ NMR $(400 \mathrm{MHz}$, $\left.\mathrm{CDCl}_{3}\right) \delta: 8.09(\mathrm{~s}, 1 \mathrm{H}$, triazole- $\mathrm{H}), 7.77(\mathrm{~s}, 1 \mathrm{H}$, triazole- $\mathrm{H})$, $7.72 \sim 7.76$ (m, 4H, ArH), 6.96 (d, $J=8.8 \mathrm{~Hz}, 2 \mathrm{H}, \mathrm{ArH})$, $6.88(\mathrm{~d}, J=8.4 \mathrm{~Hz}, 2 \mathrm{H}, \mathrm{ArH}), 5.23 \sim 5.38(\mathrm{~m}, 2 \mathrm{H}$, $\left.\mathrm{OCOCH}_{2}\right), 5.07 \sim 5.10\left(\mathrm{~m}, 2 \mathrm{H}, \mathrm{OCOCH}_{2}\right), 4.91(\mathrm{~s}, 1 \mathrm{H}$, $7 \beta-\mathrm{H}), 4.61 \sim 4.66(\mathrm{~m}, 1 \mathrm{H}, 3 \beta-\mathrm{H}), 3.84\left(\mathrm{~s}, 3 \mathrm{H}, \mathrm{ArOCH}_{3}\right)$, $3.80\left(\mathrm{~s}, 3 \mathrm{H}, \mathrm{ArOCH}_{3}\right), 3.68$ (s, 3H, $\left.\mathrm{COOCH}_{3}\right), 0.89$ (s, $3 \mathrm{H}$, $\left.19-\mathrm{CH}_{3}\right), 0.84\left(\mathrm{~d}, J=6.4 \mathrm{~Hz}, 3 \mathrm{H}, 21-\mathrm{CH}_{3}\right), 0.59$ (s, $3 \mathrm{H}$, 18- $\mathrm{CH}_{3}$ ); IR (KBr) $v: 2943,2869,1736,1618,1561,1500$, 1459, 1364, 1249, 1226, 1175, 1032, $836 \mathrm{~cm}^{-1}$; ESI-MS $m / z(\%): 837\left[(\mathrm{M}+\mathrm{H})^{+}, 100\right]$. Anal. calcd for $\mathrm{C}_{47} \mathrm{H}_{60} \mathrm{~N}_{6} \mathrm{O}_{8}$ : C 67.44, H 7.23, N 10.04; found C 67.40, H 7.21, N 10.07.

分子钳 5d: 白色固体, 产率 84\%. m.p. 117 119 ${ }^{\circ} \mathrm{C} ;[\alpha]_{\mathrm{D}}^{20}-66.4\left(c \quad 0.13, \mathrm{CH}_{2} \mathrm{Cl}_{2}\right)$; ${ }^{1} \mathrm{H}$ NMR $(400 \mathrm{MHz}$, $\left.\mathrm{CDCl}_{3}\right) \delta: 8.12(\mathrm{~s}, 1 \mathrm{H}$, triazole- $\mathrm{H}), 7.80(\mathrm{~s}, 1 \mathrm{H}$, triazole- $\mathrm{H})$, $7.10 \sim 7.28(\mathrm{~m}, 6 \mathrm{H}, \mathrm{ArH}), 6.69(\mathrm{~d}, J=7.2 \mathrm{~Hz}, 1 \mathrm{H}, \mathrm{ArH})$, $6.62(\mathrm{~d}, J=7.2 \mathrm{~Hz}, 1 \mathrm{H}, \operatorname{ArH}), 5.23 \sim 5.38(\mathrm{~m}, 2 \mathrm{H}$, $\left.\mathrm{OCOCH}_{2}\right), 5.01 \sim 5.10\left(\mathrm{~m}, 2 \mathrm{H}, \mathrm{OCOCH}_{2}\right), 4.89(\mathrm{~s}, 1 \mathrm{H}$, $7 \beta-\mathrm{H}), 4.61 \sim 4.66(\mathrm{~m}, 1 \mathrm{H}, 3 \beta-\mathrm{H}), 3.68\left(\mathrm{~s}, 3 \mathrm{H}, \mathrm{COOCH}_{3}\right)$, $0.88\left(\mathrm{~s}, 3 \mathrm{H}, 19-\mathrm{CH}_{3}\right), 0.84\left(\mathrm{~d}, J=6.4 \mathrm{~Hz}, 3 \mathrm{H}, 21-\mathrm{CH}_{3}\right)$, 0.58 (s, 3H, 18- $\mathrm{CH}_{3}$ ); IR (KBr) v: 3449, 3370, 2942, 2869, 1736, 1619, 1591, 1458, 1433, 1364, 1274, 1224, 1139, 1045, $783 \mathrm{~cm}^{-1}$; ESI-MS $m / z(\%): 935\left[(\mathrm{M}+\mathrm{H})^{+}, 100\right]$. 
Anal. calcd for $\mathrm{C}_{45} \mathrm{H}_{54} \mathrm{Br}_{2} \mathrm{~N}_{6} \mathrm{O}_{6}$ : C 57.82, H 5.82, N 8.99; found C 57.73, H 5.84, N 9.02.

分子钳 5e: 白色固体, 产率 $87 \%$. m.p. 110 112 ${ }^{\circ} \mathrm{C} .[\alpha]_{\mathrm{D}}^{20}-59.7\left(c \quad 0.16, \mathrm{CH}_{2} \mathrm{Cl}_{2}\right) ;{ }^{1} \mathrm{H}$ NMR $(400 \mathrm{MHz}$, $\left.\mathrm{CDCl}_{3}\right) \delta: 8.18(\mathrm{~s}, 1 \mathrm{H}$, triazole- $\mathrm{H}), 7.86(\mathrm{~s}, 1 \mathrm{H}$, triazole- $\mathrm{H})$, $7.66 \sim 7.71(\mathrm{~m}, 4 \mathrm{H}, \mathrm{ArH}), 7.56(\mathrm{~d}, J=8.4 \mathrm{~Hz}, 2 \mathrm{H}, \mathrm{ArH})$, $7.47(\mathrm{~d}, J=8.4 \mathrm{~Hz}, 2 \mathrm{H}, \operatorname{ArH}), 5.25 \sim 5.41(\mathrm{~m}, 2 \mathrm{H}$, $\left.\mathrm{OCOCH}_{2}\right), 5.05 \sim 5.17\left(\mathrm{~m}, 2 \mathrm{H}, \mathrm{OCOCH}_{2}\right), 4.91(\mathrm{~s}, 1 \mathrm{H}$, $7 \beta-\mathrm{H}), 4.62 \sim 4.67(\mathrm{~m}, 1 \mathrm{H}, 3 \beta-\mathrm{H}), 3.69\left(\mathrm{~s}, 3 \mathrm{H}, \mathrm{COOCH}_{3}\right)$, $2.40\left(\mathrm{~s}, 3 \mathrm{H}, \mathrm{Ar}-\mathrm{CH}_{3}\right), 2.38$ (s, $\left.3 \mathrm{H}, \mathrm{Ar}-\mathrm{CH}_{3}\right), 0.89$ (s, $3 \mathrm{H}$, $\left.19-\mathrm{CH}_{3}\right), 0.85$ (d, $\left.J=6.4 \mathrm{~Hz}, 3 \mathrm{H}, 21-\mathrm{CH}_{3}\right), 0.59$ (s, $3 \mathrm{H}$, $\left.18-\mathrm{CH}_{3}\right)$; IR (KBr) v: 2944, 2869, 1737, 1459, 1271, 1226, 1139, 1068, 1010, $974 \mathrm{~cm}^{-1}$; ESI-MS $m / z$ (\%): 805 [(M+ $\left.\mathrm{H})^{+}, 100\right]$. Anal. calcd for $\mathrm{C}_{47} \mathrm{H}_{60} \mathrm{~N}_{6} \mathrm{O}_{6}: \mathrm{C} 70.12, \mathrm{H} 7.51, \mathrm{~N}$ 10.44; found C 70.21, H 7.53, N 10.40.

分子钳 5f: 白色固体，产率 $91 \%$. m.p. $159 \sim 161{ }^{\circ} \mathrm{C}$; $[\alpha]_{\mathrm{D}}^{20}-83.7\left(c \quad 0.19, \mathrm{CH}_{2} \mathrm{Cl}_{2}\right) ;{ }^{1} \mathrm{H}$ NMR $(400 \mathrm{MHz}$, $\left.\mathrm{CDCl}_{3}\right) \delta: 8.14(\mathrm{~s}, 1 \mathrm{H}$, triazole- $\mathrm{H}), 7.84(\mathrm{~s}, 1 \mathrm{H}$, triazole- $\mathrm{H})$, $7.77 \sim 7.83(\mathrm{~m}, 4 \mathrm{H}, \mathrm{ArH}), 7.13(\mathrm{t}, J=8.6 \mathrm{~Hz}, 2 \mathrm{H}, \mathrm{ArH})$, $7.05(\mathrm{t}, J=8.6 \mathrm{~Hz}, 2 \mathrm{H}, \operatorname{ArH}), 5.25 \sim 5.41(\mathrm{~m}, 2 \mathrm{H}$, $\left.\mathrm{OCOCH}_{2}\right), 5.06 \sim 5.18\left(\mathrm{~m}, 2 \mathrm{H}, \mathrm{OCOCH}_{2}\right), 4.92(\mathrm{~s}, 1 \mathrm{H}$, $7 \beta-\mathrm{H}), 4.62 \sim 4.68(\mathrm{~m}, 1 \mathrm{H}, 3 \beta-\mathrm{H}), 3.69\left(\mathrm{~s}, 3 \mathrm{H}, \mathrm{COOCH}_{3}\right)$, 0.90 (s, 3H, 19- $\left.\mathrm{CH}_{3}\right), 0.85$ (d, $J=6.4 \mathrm{~Hz}, 3 \mathrm{H}, 21-\mathrm{CH}_{3}$ ), 0.60 (s, 3H, 18- $\mathrm{CH}_{3}$ ); IR (KBr) v: 2945, 2869, 1741, 1561, 1499, 1460, 1362, 1275, 1223, 1158, 1045, 977, 842, 795 $\mathrm{cm}^{-1}$; ESI-MS $m / z(\%): 845\left[(\mathrm{M}+\mathrm{H})^{+}, 100\right]$. Anal. calcd for $\mathrm{C}_{45} \mathrm{H}_{54} \mathrm{Cl}_{2} \mathrm{~N}_{6} \mathrm{O}_{6}$ : C 63.90, $\mathrm{H}$ 6.43, N 9.94; found $\mathrm{C}$ 63.98, H 6.41, N 9.97.

分子钳 5g: 白色固体, 产率 92\%. m.p. 100 102 ${ }^{\circ} \mathrm{C} ;[\alpha]_{\mathrm{D}}^{20}-156.3\left(c 0.16, \mathrm{CH}_{2} \mathrm{Cl}_{2}\right) ;{ }^{1} \mathrm{H}$ NMR $(400 \mathrm{MHz}$, $\left.\mathrm{CDCl}_{3}\right) \delta: 8.16(\mathrm{~s}, 1 \mathrm{H}$, triazole- $\mathrm{H}), 7.80(\mathrm{~s}, 1 \mathrm{H}$, triazole- $\mathrm{H})$, $7.72 \sim 7.76$ (m, 4H, ArH), 7.26 7.28 (m, 2H, ArH), 7.18 (d, $J=8.0 \mathrm{~Hz}, 2 \mathrm{H}, \mathrm{ArH}), 5.23 \sim 5.39\left(\mathrm{~m}, 2 \mathrm{H}, \mathrm{OCOCH}_{2}\right)$, $5.02 \sim 5.13\left(\mathrm{~m}, 2 \mathrm{H}, \mathrm{OCOCH}_{2}\right), 4.92(\mathrm{~s}, 1 \mathrm{H}, 7 \beta-\mathrm{H}), 4.60 \sim$ $4.66(\mathrm{~m}, 1 \mathrm{H}, 3 \beta-\mathrm{H}), 3.69\left(\mathrm{~s}, 3 \mathrm{H}, \mathrm{COOCH}_{3}\right), 2.61 \sim 2.71$ (m, $\left.4 \mathrm{H}, \mathrm{Ar}-\mathrm{CH}_{2} \times 2\right), 1.22 \sim 1.28\left(\mathrm{~m}, 6 \mathrm{H}, \mathrm{Ar}-\mathrm{CH}_{2} \mathrm{CH}_{3} \times 2\right)$, 0.88 (s, 3H, 19- $\left.\mathrm{CH}_{3}\right), 0.82\left(\mathrm{~d}, J=6.4 \mathrm{~Hz}, 3 \mathrm{H}, 21-\mathrm{CH}_{3}\right)$, 0.59 (s, 3H, 18- $\mathrm{CH}_{3}$ ); IR (KBr) v: 2945, 2869, 1741, 1487, 1459, 1272, 1221, 1093, 1044, $976 \mathrm{~cm}^{-1}$; ESI-MS m/z (\%): $833\left[(\mathrm{M}+\mathrm{H})^{+}, 100\right]$. Anal. calcd for $\mathrm{C}_{49} \mathrm{H}_{64} \mathrm{C}_{12} \mathrm{~N}_{6} \mathrm{O}_{6}: \mathrm{C}$ 70.65, H 7.74, N 10.09; found C 70.55, H 7.71, N10.12.

分子钳 5h: 白色固体, 产率 $88 \%$. m.p. $98 \sim 100{ }^{\circ} \mathrm{C}$; $[\alpha]_{\mathrm{D}}^{20}-56.3\left(\right.$ c $\left.0.21, \mathrm{CH}_{2} \mathrm{Cl}_{2}\right) ;{ }^{1} \mathrm{H}$ NMR $(400 \mathrm{MHz}$, $\left.\mathrm{CDCl}_{3}\right) \delta: 8.13(\mathrm{~s}, 1 \mathrm{H}$, triazole- $\mathrm{H}), 7.80(\mathrm{~s}, 1 \mathrm{H}$, triazole- $\mathrm{H})$, 7.71 (t, $J=8.6 \mathrm{~Hz}, 4 \mathrm{H}, \mathrm{ArH}), 7.24$ (d, $J=7.6 \mathrm{~Hz}, 2 \mathrm{H}$,
$\operatorname{ArH}), 7.16(\mathrm{~d}, J=7.6 \mathrm{~Hz}, 2 \mathrm{H}, \mathrm{ArH}), 5.23 \sim 5.39(\mathrm{~m}, 2 \mathrm{H}$, $\left.\mathrm{OCOCH}_{2}\right), 5.01 \sim 5.14\left(\mathrm{~m}, 2 \mathrm{H}, \mathrm{OCOCH}_{2}\right), 4.91(\mathrm{~s}, 1 \mathrm{H}$, $7 \beta-\mathrm{H}), 4.60 \sim 4.66(\mathrm{~m}, 1 \mathrm{H}, 3 \beta-\mathrm{H}), 3.68\left(\mathrm{~s}, 3 \mathrm{H}, \mathrm{COOCH}_{3}\right)$, 2.39 (s, $\left.3 \mathrm{H}, \mathrm{ArCH}_{3}\right), 2.34$ (s, 3H, $\left.\mathrm{ArCH}_{3}\right), 0.88$ (s, 3H, $\left.19-\mathrm{CH}_{3}\right), 0.84\left(\mathrm{~d}, J=6.4 \mathrm{~Hz}, 3 \mathrm{H}, 21-\mathrm{CH}_{3}\right), 0.59$ (s, 3H, $18-\mathrm{CH}_{3}$ ); IR (KBr) v: 2943, 2868, 1741, 1459, 1379, 1362, 1274, 1215, 1138, 1044, $972 \mathrm{~cm}^{-1}$; ESI-MS $m / z$ (\%): 813 $\left[(\mathrm{M}+\mathrm{H})^{+}, 100\right]$. Anal. calcd for $\mathrm{C}_{45} \mathrm{H}_{54} \mathrm{~F}_{2} \mathrm{~N}_{6} \mathrm{O}_{6}: \mathrm{C} 66.48, \mathrm{H}$ 6.70, N 10.34; found C 66.38, H 6.730, N 10.31.

分子钳 5i: 白色固体, 产率 $87 \%$. m.p. $94 \sim 96{ }^{\circ} \mathrm{C}$;

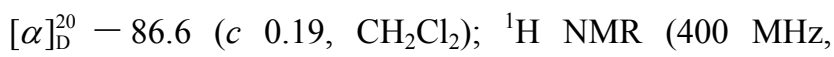
$\left.\mathrm{CDCl}_{3}\right) \delta: 8.16(\mathrm{~s}, 1 \mathrm{H}$, triazole- $\mathrm{H}), 7.80(\mathrm{~s}, 1 \mathrm{H}$, triazole- $\mathrm{H})$, 7.74 (t, $J=8.6 \mathrm{~Hz}, 4 \mathrm{H}, \mathrm{ArH}), 7.24$ (d, $J=8 \mathrm{~Hz}, 2 \mathrm{H}, \mathrm{ArH})$, $7.16(\mathrm{~d}, J=8 \mathrm{~Hz}, 2 \mathrm{H}, \operatorname{ArH}), 5.23 \sim 5.39(\mathrm{~m}, 2 \mathrm{H}$, $\left.\mathrm{OCOCH}_{2}\right), 5.02 \sim 5.13\left(\mathrm{~m}, 2 \mathrm{H}, \mathrm{OCOCH}_{2}\right), 4.91(\mathrm{~s}, 1 \mathrm{H}$, $7 \beta-\mathrm{H}), 4.62 \sim 4.64(\mathrm{~m}, 1 \mathrm{H}, 3 \beta-\mathrm{H}), 3.68\left(\mathrm{~s}, 3 \mathrm{H}, \mathrm{COOCH}_{3}\right)$, $2.56 \sim 2.64\left(\mathrm{~m}, 4 \mathrm{H}, \mathrm{Ar}-\mathrm{CH}_{2} \times 2\right), 1.65 \sim 1.69(\mathrm{~m}, 4 \mathrm{H}$, $\left.\mathrm{Ar}-\mathrm{CH}_{2} \mathrm{CH}_{2} \times 2\right), 0.93 \sim 1.04\left(\mathrm{~m}, 6 \mathrm{H}, \mathrm{ArC}_{2} \mathrm{H}_{4} \mathrm{CH}_{3} \times 2\right)$, $0.88\left(\mathrm{~s}, 3 \mathrm{H}, 19-\mathrm{CH}_{3}\right), 0.83\left(\mathrm{~d}, J=6.4 \mathrm{~Hz}, 3 \mathrm{H}, 21-\mathrm{CH}_{3}\right)$, 0.58 (s, 3H, 18- $\left.\mathrm{CH}_{3}\right)$; IR (KBr) v: 2955, 2870, 1741, 1460, 1379, 1363, 1275, 1227, 1044, $976 \mathrm{~cm}^{-1}$; ESI-MS $m / z(\%)$ : $861\left[(\mathrm{M}+\mathrm{H})^{+}, 100\right]$. Anal. calcd for $\mathrm{C}_{51} \mathrm{H}_{68} \mathrm{~N}_{6} \mathrm{O}_{6}: \mathrm{C} 71.13$, H 7.96, N 9.76; found C 71. 08, H 7.94, N 9.74.

分子钳 5j: 黄色固体, 产率 $89 \%$. m.p. $138 \sim 140{ }^{\circ} \mathrm{C}$; $[\alpha]_{\mathrm{D}}^{20}-30.3\left(c \quad 0.15, \mathrm{CH}_{2} \mathrm{Cl}_{2}\right) ;{ }^{1} \mathrm{H}$ NMR $(400 \mathrm{MHz}$, $\left.\mathrm{CDCl}_{3}\right) \delta: 8.31(\mathrm{~s}, 1 \mathrm{H}$, triazole- $\mathrm{H}), 8.30(\mathrm{~s}, 1 \mathrm{H}$, triazole- $\mathrm{H})$, 8.28 (s, 1H, ArH), 8.25 (s, 1H, ArH), 8.23 (s, 1H, ArH), 8.07 (s, $1 \mathrm{H}, \mathrm{ArH}), 7.99 \sim 8.02(\mathrm{~m}, 4 \mathrm{H}, \mathrm{ArH}), 5.14 \sim 5.46$ $\left(\mathrm{m}, 4 \mathrm{H}, \mathrm{OCOCH}_{2} \times 2\right), 4.96(\mathrm{~s}, 1 \mathrm{H}, 7 \beta-\mathrm{H}), 4.65 \sim 4.71(\mathrm{~m}$, $1 \mathrm{H}, 3 \beta-\mathrm{H}), 3.68\left(\mathrm{~s}, 3 \mathrm{H}, \mathrm{COOCH}_{3}\right), 0.91\left(\mathrm{~s}, 3 \mathrm{H}, 19-\mathrm{CH}_{3}\right)$, 0.85 (d, $\left.J=6.4 \mathrm{~Hz}, 3 \mathrm{H}, 21-\mathrm{CH}_{3}\right), 0.61$ (s, $\left.3 \mathrm{H}, 18-\mathrm{CH}_{3}\right)$; IR (KBr) $v$ : 2944, 2869, 1742, 1607, 1521, 1460, 1337, 1222, 1110, 1044, 976, 853, $756 \mathrm{~cm}^{-1}$; ESI-MS $\mathrm{m} / z$ (\%): 889 $\left[(\mathrm{M}+\mathrm{Na})^{+}, 100\right]$. Anal. calcd for $\mathrm{C}_{45} \mathrm{H}_{54} \mathrm{~N}_{8} \mathrm{O}_{10}: \mathrm{C} 62.34, \mathrm{H}$ 6.28, N 12.92; found C 62.28, H 6.26, N 12.88.

分子钳 5k: 黄色固体, 产率 92\%. m.p. 90 92 ${ }^{\circ} \mathrm{C}$; $[\alpha]_{\mathrm{D}}^{20}-168.2\left(c \quad 0.14, \mathrm{CH}_{2} \mathrm{Cl}_{2}\right) ;{ }^{1} \mathrm{H}$ NMR $(400 \mathrm{MHz}$, $\left.\mathrm{CDCl}_{3}\right) \delta: 10.45(\mathrm{~s}, 2 \mathrm{H}, \mathrm{CHO} \times 2), 8.08(\mathrm{~s}, 1 \mathrm{H}$, triazole- $\mathrm{H})$, $7.86(\mathrm{~s}, 1 \mathrm{H}$, triazole-H), $7.81 \sim 7.84(\mathrm{~m}, 2 \mathrm{H}, \mathrm{ArH}), 7.53 \sim$ $7.58(\mathrm{~m}, 2 \mathrm{H}, \mathrm{ArH}), 7.14 \sim 7.20$ (m, 2H, ArH), $7.04 \sim 7.09$ (m, $2 \mathrm{H}, \mathrm{ArH}), 5.15 \sim 5.45\left(\mathrm{~m}, 8 \mathrm{H}, \mathrm{OCH}_{2} \times 2, \mathrm{OCOCH}_{2} \times\right.$ 2), $4.99(\mathrm{~s}, 1 \mathrm{H}, 7 \beta-\mathrm{H}), 4.59 \sim 4.64(\mathrm{~m}, 1 \mathrm{H}, 3 \beta-\mathrm{H}), 3.67(\mathrm{~s}$, $\left.3 \mathrm{H}, \mathrm{COOCH}_{3}\right), 0.91\left(\mathrm{~s}, 3 \mathrm{H}, 19-\mathrm{CH}_{3}\right), 0.89(\mathrm{~d}, J=8.4 \mathrm{~Hz}$, $\left.3 \mathrm{H}, 21-\mathrm{CH}_{3}\right), 0.63\left(\mathrm{~s}, 3 \mathrm{H}, 18-\mathrm{CH}_{3}\right) ; \mathrm{IR}(\mathrm{KBr}) v: 2945$, 2869, 1740, 1690, 1594, 1478, 1458, 1288, 1213, 1163 , 
1047, 993, $761 \mathrm{~cm}^{-1}$; ESI-MS m/z (\%): $915\left[(\mathrm{M}+\mathrm{Na})^{+}\right.$, 100]. Anal. calcd for $\mathrm{C}_{49} \mathrm{H}_{60} \mathrm{~N}_{6} \mathrm{O}_{10}: \mathrm{C} 65.90, \mathrm{H} 6.77, \mathrm{~N}$ 9.41; found C 65.79, H 6.79, N 9.43.

分子钳 5l: 黄色固体, 产率 89\%. m.p. 103 $105{ }^{\circ} \mathrm{C}$, $[\alpha]_{\mathrm{D}}^{20}-49.2\left(c \quad 0.12, \mathrm{CH}_{2} \mathrm{Cl}_{2}\right) ;{ }^{1} \mathrm{H} \mathrm{NMR}(400 \mathrm{MHz}$, $\left.\mathrm{CDCl}_{3}\right) \delta: 10.87$ (s, 1H, CHO), 10.85 (s, 1H, CHO), 9.21 (s, 1H, ArH), 9.19 (s, 1H, ArH), $8.10(\mathrm{~s}, 1 \mathrm{H}$, triazole-H), $7.98 \sim 8.01(\mathrm{~m}, 2 \mathrm{H}, \mathrm{ArH}), 7.83(\mathrm{~s}, 1 \mathrm{H}$, triazole-H), $7.73(\mathrm{~d}$, $J=8.4 \mathrm{~Hz}, 2 \mathrm{H}, \mathrm{ArH}), 7.58 \sim 7.62(\mathrm{~m}, 2 \mathrm{H}, \mathrm{ArH}), 7.36 \sim$ $7.43(\mathrm{~m}, 4 \mathrm{H}, \mathrm{ArH}), 5.18 \sim 5.45\left(\mathrm{~m}, 8 \mathrm{H}, \mathrm{OCH}_{2} \times 2\right.$, $\left.\mathrm{OCOCH}_{2} \times 2\right), 4.97(\mathrm{~s}, 1 \mathrm{H}, 7 \beta-\mathrm{H}), 4.64 \sim 4.67(\mathrm{~m}, 1 \mathrm{H}$, $3 \beta-\mathrm{H}), 3.66$ (s, $\left.3 \mathrm{H}, \mathrm{COOCH}_{3}\right), 0.89$ (s, 3H, 19- $\left.\mathrm{CH}_{3}\right), 0.86$ (d, $\left.J=6 \mathrm{~Hz}, 3 \mathrm{H}, 21-\mathrm{CH}_{3}\right), 0.61\left(\mathrm{~s}, 3 \mathrm{H}, 18-\mathrm{CH}_{3}\right)$; IR (KBr) v: 2943, 2871, 1736, 1619, 1591, 1510, 1460, 1437, 1365, 1342, 1269, 1213, 1154, 1048, 1019, $814 \mathrm{~cm}^{-1}$; ESI-MS $m / z(\%): 1015\left[(\mathrm{M}+\mathrm{Na})^{+}, 100\right]$. Anal. calcd for $\mathrm{C}_{57} \mathrm{H}_{64^{-}}$ $\mathrm{N}_{6} \mathrm{O}_{10}$ : C 68.93, H 6.50, N 8.46; found C 68.84, H 6.48, N 8.42 .

\section{4 识别性能测试}

以氯仿-甲醇混合液作溶剂, 固定主体分子钳的浓 度在 $1 \times 10^{-5} \sim 10 \times 10^{-5} \mathrm{~mol} \cdot \mathrm{L}^{-1}$ 之间(由主体摩尔消光 系数的大小决定所配溶液浓度的大小), 不断加入客体 分子, 其浓度在 $10^{-4} \sim 10^{-3} \mathrm{~mol} \cdot \mathrm{L}^{-1}$ 间变化, 测定各组 配合物溶液的吸光度值, 为消除客体化合物在紫外区的 干扰，采用相同浓度的客体氯仿液作参比，同时为排除 体积变化对吸光度的影响, 主体中加入客体化合物的总 体积不超过 $100 \mu \mathrm{L}$. 测定温度控制在 $(25 \pm 0.1){ }^{\circ} \mathrm{C}$.

\section{2 结果与讨论}

\section{1 目标分子钳的合成}

在 Click reaction 这一步, 我们以钳形受体 5a 的合 成为例, 详细考察了溶剂、温度以及催化剂浓度等对反 应的影响，其结果如表 1 、表 2 和表 3 所示.

表 1 溶剂对分子钳 5a 产率的影响

Table 1 The effect of solvent on the yields of compound 5a

\begin{tabular}{lcc}
\hline 溶剂 & 温度 $/{ }^{\circ} \mathrm{C}$ & 产率 ${ }^{a} / \%$ \\
\hline $\mathrm{DMSO}$ & 50 & 33 \\
$\mathrm{DMF}$ & 50 & 38 \\
$\mathrm{THF}$ & 50 & 45 \\
$t$-BuOH & 50 & 93 \\
\hline${ }^{a}$ 分离纯化的产率. & &
\end{tabular}

由表 1 和表 2 可以看出, 选用 $t-\mathrm{BuOH}$ 作为溶剂, 在 $50{ }^{\circ} \mathrm{C}$ 反应, 反应产率最高. 由表 3 可以看出, 当催化剂 为 $10 \mathrm{~mol} \%$ 的 $\mathrm{CuI}$ 和 $40 \mathrm{~mol} \%$ 的 $\mathrm{Et}_{3} \mathrm{~N}$ 时, 产率最高. 反 应时间对产率的影响也非常明显, 整个实验过程通过
表 2 温度对分子钳 $5 \mathrm{a}$ 产率的影响

Table 2 The effect of temperature on the yields of compound 5a

\begin{tabular}{ccc}
\hline 溶剂 & 温度 $/{ }^{\circ} \mathrm{C}$ & 产率 $^{b} / \%$ \\
\hline$t-\mathrm{BuOH}$ & 0 & 30 \\
$t-\mathrm{BuOH}$ & 25 & 61 \\
$t-\mathrm{BuOH}$ & 50 & 93 \\
$t-\mathrm{BuOH}$ & 75 & 79 \\
\hline${ }^{b}$ 分离纯化的产率. & &
\end{tabular}

表 3 催化剂浓度对分子钳 $5 \mathbf{a}$ 产率的影响

Table 3 The effect of concentration of catalysts on the yields of compound $\mathbf{5 a}$

\begin{tabular}{|c|c|c|}
\hline \multicolumn{2}{|c|}{ 催化剂 $/ \mathrm{mol} \%$} & \multirow{2}{*}{ 产率 ${ }^{c} / \%$} \\
\hline $\mathrm{CuI}$ & $\mathrm{Et}_{3} \mathrm{~N}$ & \\
\hline 10 & 0 & $<10$ \\
\hline 10 & 20 & 55 \\
\hline 10 & 40 & 93 \\
\hline 10 & 60 & 83 \\
\hline 0 & 40 & $<10$ \\
\hline 5 & 40 & 62 \\
\hline 15 & 40 & 85 \\
\hline
\end{tabular}

TLC 监测，发现时间过短，反应不够完全，不仅产率很 低而且副产物比较多. 综合上述实验结果找到了合成目 标物的最佳条件是: $t-\mathrm{BuOH}$ 为溶剂, $40 \mathrm{~mol} \%$ 三乙胺, $10 \mathrm{~mol} \% \mathrm{CuI}$, 在 $50{ }^{\circ} \mathrm{C}$, 油浴加热反应 $10 \sim 30 \mathrm{~h}$.

\section{2 目标物 $5 \mathrm{a} \sim 5 \mathrm{I}$ 的结构确证}

目标物 $\mathbf{5 a} \sim \mathbf{5 j}$ : 在 ${ }^{1} \mathrm{H} \mathrm{NMR}$ 谱图中, $\delta 8.32 \sim 7.77$ $\mathrm{ppm}$ 为三唑环上的 $\mathrm{C}-\mathrm{H}$ 的化学位移, 而且其质子数为 两个, 与连接在两个手臂上的三唑环上的碳氢质子数相 符合, $\delta 8.28 \sim 6.62$ 左右的多重峰为侨联在两手臂上芳 环的化学位移; 在 IR 谱图中, 在 $1700 \mathrm{~cm}^{-1}$ 附近出现甲 酯羰基 $(\mathrm{C}=\mathrm{O})$ 的伸缩振动吸收峰; 从质谱图中可见，所 有目标化合物均能给出分子离子峰, 其 $m / z$ 与相应分子 式的分子量是一致的. 元素分析结果表明, 所有化合物 的组成与其实验式相符. 目标物 $\mathbf{5 k}, \mathbf{5 l}$ : 在 ${ }^{1} \mathrm{H}$ NMR 谱 图中, $\delta 10.87 \sim 10.45$ 的单峰为芳环上的醛基结构, 其质 子数与结构一致, $\delta 8.10 \sim 7.83$ 的单峰为三唑环上的 $\mathrm{C}-$ $\mathrm{H}$ 的化学位移, 而且其质子数为两个, 与连接在两个手 臂上的三唑环上的碳氢质子数相符合, $\delta 7.84 \sim 7.04$ 的 多重峰为桥联在两手臂上芳环的化学位移. 综上所述, 化合物 $\mathbf{5 a} \sim \mathbf{5 l}$ 所有光谱和元素分析数据与其结构式符 合，表明所合成的化合物为目标化合物.

\section{3 主客体配合物的形成及其化学计量}

在紫外光谱滴定实验中，固定主体分子钳 $(\mathrm{H})$ 浓度， 向主体溶液中不断加入不同浓度的客体阳离子 $(\mathrm{G})$ 溶液, 随着客体阳离子的不断加入, 主体生色团的最大吸收呈 
规律性下降, 表明主客体分子之间并发生了识别配合作 用(图 1). 由图 2 的 Job's plot 可知, 主体分子钳 $5 \mathbf{k}$ 与客 体阳离子形成配合物的配合比例是 $1: 1$. 对所考察的 客体阳离子, 根据 Hildebrand-Benesi ${ }^{[23]}$ 方程(1), 如若主 体与客体之间形成了 $1: 1$ 型超分子配合物, 则 $1 / \Delta A$ 对 $1 /[G]_{0}$ 作图, 给出良好的线性关系. 由图 3 良好的线性 关系可得知, 被考察的分子钳 $\mathbf{5 k}$ 与客体金属阳离子形 成了 $1: 1$ 型超分子配合物. 由直线的截距和斜率所得 的数据可计算出配合物的结合常数 $\left(K_{\mathrm{a}}\right)$ 和自由能变化 $\left(-\Delta G^{0}\right)$. 所考察的主体分子钳 $5 \mathbf{a}, 5 \mathbf{h}, 5 \mathbf{k}, 5 \mathbf{1}$ 的结合常数 $\left(K_{\mathrm{a}}\right)$ 和自由能变化 $\left(-\Delta G^{0}\right)$ 见表 4 .

$$
\frac{1}{\Delta A}=\frac{1}{\alpha K_{\mathrm{a}}[\mathrm{G}]_{0}}+\frac{1}{\alpha}
$$

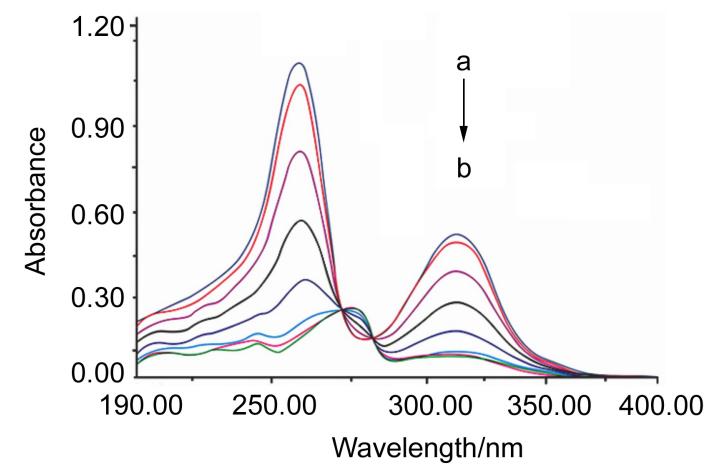

图 1 分子钳 $\mathbf{5 k}\left(2.4 \times 10^{-5} \mathrm{~mol} \cdot \mathrm{L}^{-1}\right)$ 中加入采离子的紫外光谱 变化图

Figure 1 UV-Vis spectra of molecular tweezer 5k $\left(2.4 \times 10^{-5}\right.$ $\mathrm{mol} \cdot \mathrm{L}^{-1}$ ) in the presence of $\mathrm{Hg}^{2+}$

(a) 0 ; (b) $3.20 \times 10^{-5}$; (c) $4.80 \times 10^{-5}$; (d) $6.40 \times 10^{-5}$; (e) $8.00 \times 10^{-5}$; (f) $9.60 \times 10^{-5}$; (g) $11.20 \times 10^{-5}$; (h) $12.80 \times 10^{-5} \mathrm{~mol} \cdot \mathrm{L}^{-1} \mathrm{Hg}^{2+}$ with $\lambda_{\text {max }}$ at $254.0 \mathrm{~nm}$

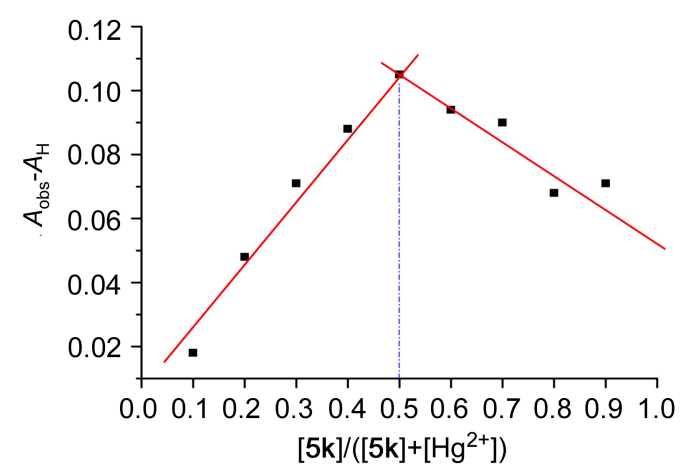

图 2 分子钳 $\mathbf{5 k}$ 与录离子形成 1: 1 型配合物的工作曲线 Figure 2 Job' plot showing $1: 1$ complex formation for compound $\mathbf{5 k}$ and $\mathrm{Hg}^{2+}$

由表 4 可看出, 这类主体对所考察的阳离子具有良 好的识别能力, 主体与底物间形成了 $1: 1$ 型超分子配 合物, 主客体在金属配合作用力、 $\pi-\pi$ stacking 作用等非

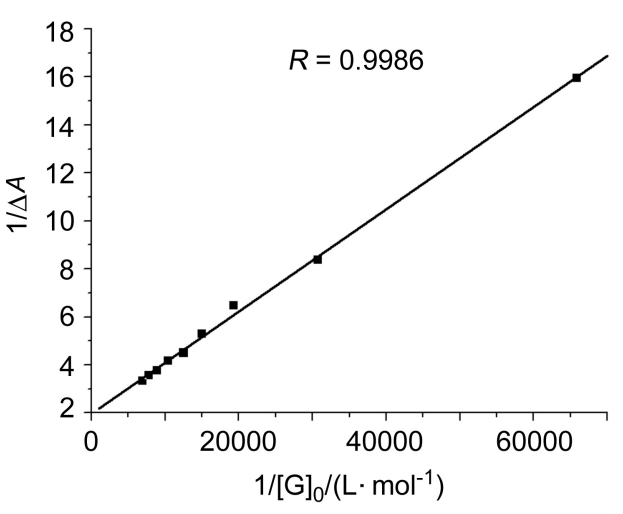

图 $325{ }^{\circ} \mathrm{C}$ 时, 在 $\mathrm{CHCl}_{3}-\mathrm{CH}_{3} \mathrm{OH}$ 中, 分子钳 $\mathbf{5 k}$ 与 $\mathrm{Hg}^{2+}$ 形成 $1: 1$ 配合物的 $1 / \Delta A$ 对 $1 /[\mathrm{G}]_{0}$ 作图

Figure 3 Typical plot of $1 / \Delta A$ versus $1 /[\mathrm{G}]_{0}$ for the $1: 1$ inclusion complex of molecular tweezer $5 \mathbf{k}$ with $\mathrm{Hg}^{2+}$ in $\mathrm{CHCl}_{3} \mathrm{CH}_{3} \mathrm{OH}$ at $25{ }^{\circ} \mathrm{C}$

表 $425{ }^{\circ} \mathrm{C}$ 下分子钳 $\mathbf{5 a}, \mathbf{5 h}, \mathbf{5 k}, \mathbf{5 l}$ 与阳离子形成包结配合物 的结合常数 $\left(K_{\mathrm{a}}\right)$ 和自由能变化 $\left(-\Delta G^{o}\right)$

Table 4 Association constants $\left(K_{\mathrm{a}}\right)$ and Gibbs free energy changes $\left(-\Delta G^{\circ}\right)$ for the inclusion complexes of cations with molecular tweezers $\mathbf{5 a}, \mathbf{5 h}, \mathbf{5 k}, \mathbf{5 l}$ in $\mathrm{CHCl}_{3} \mathrm{CH}_{3} \mathrm{OH}(V: V=7$ : 2) at $25{ }^{\circ} \mathrm{C}$

\begin{tabular}{|c|c|c|c|}
\hline Host & Guest & $K_{\mathrm{a}} /\left(\mathrm{L} \cdot \mathrm{mol}^{-1}\right)$ & $-\Delta G^{\mathrm{o}} /\left(\mathrm{kJ} \cdot \mathrm{mol}^{-1}\right)$ \\
\hline \multirow{4}{*}{$5 \mathbf{a}$} & $\mathrm{Hg}^{2+}$ & 896.521 & 16.82 \\
\hline & $\mathrm{Cu}^{2+}$ & 281.633 & 13.95 \\
\hline & $\mathrm{Mg}^{2+}$ & 106.203 & 11.54 \\
\hline & $\mathrm{Pb}^{2+}$ & 512.411 & 15.43 \\
\hline \multirow{4}{*}{$5 h$} & $\mathrm{Hg}^{2+}$ & 3761.06 & 20.40 \\
\hline & $\mathrm{Cu}^{2+}$ & 424.063 & 14.99 \\
\hline & $\mathrm{Mg}^{2+}$ & 539.343 & 15.58 \\
\hline & $\mathrm{Pb}^{2+}$ & 107.032 & 11.58 \\
\hline \multirow{4}{*}{$5 \mathrm{k}$} & $\mathrm{Hg}^{2+}$ & 8328.08 & 22.37 \\
\hline & $\mathrm{Cu}^{2+}$ & 434.191 & 15.05 \\
\hline & $\mathrm{Mg}^{2+}$ & 2524.97 & 19.41 \\
\hline & $\mathrm{Pb}^{2+}$ & 626.964 & 15.96 \\
\hline \multirow{4}{*}{51} & $\mathrm{Hg}^{2+}$ & 6565.87 & 21.78 \\
\hline & $\mathrm{Cu}^{2+}$ & 462.512 & 15.20 \\
\hline & $\mathrm{Mg}^{2+}$ & 27.2301 & 8.890 \\
\hline & $\mathrm{Pb}^{2+}$ & 139.532 & 12.23 \\
\hline
\end{tabular}

共价键力的协同作用下进行识别配合. 其次，上述分子 钳受体对所考察的阳离子的识别性能具有一定的相似 性，被考察的分子钳受体对录离子的识别效果好于对其 他阳离子的识别效果. 这可能由于这些钳形受体与录离 子在尺寸、形状上的匹配比其它金属阳离子匹配好, 故 对录离子显示出了更好的选择性. 分子钳对客体所表现 出的不同程度的识别效果, 表明了受体的微环境不同, 可造成其对客体识别能力的差异. 


\section{4 计算机分子模拟}

我们利用计算机 Chem 3D 程序模拟研究了主客体 间的配合作用，以期得到主客体发生配合作用时的构 象. 经分子模拟，表明受体 5a $\sim 51$ 具有多种构象异构体. 以受体 $5 \mathbf{k}$ 为例, 当其处于最低能量构象时, 受体的钳形 构象如图 4 所示, 图 5 是它与客体录离子配合物的最低 能量构象. 从图 5 中可看出, 录离子能较好的嵌入主体 裂穴, 诱导其产生配合作用.

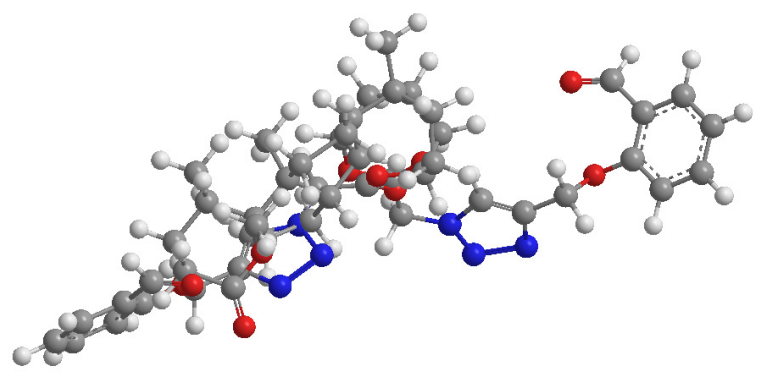

图 4 分子钳 $5 \mathbf{k}$ 的最低能量构象

Figure 4 Minimum energy conformation of molecular tweezer $5 \mathbf{k}$

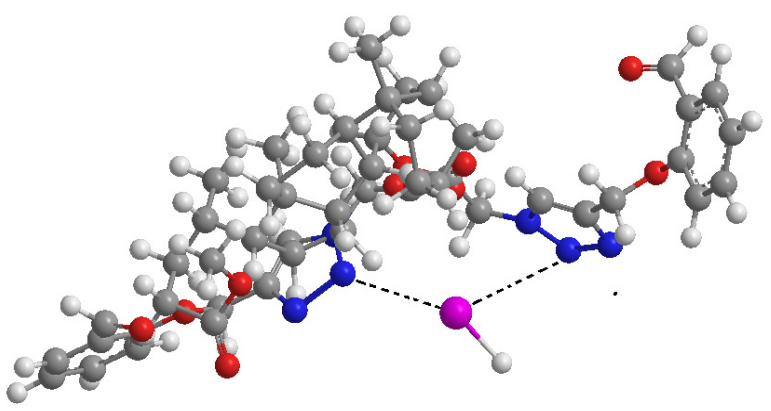

图 5 分子钳 $5 \mathrm{k}$ 与录离子配合物的最低能量构象

Figure 5 Minimum energy conformation for the inclusion complex of molecular tweezer $\mathbf{5} \mathbf{k}$ with $\mathrm{Hg}^{2+}$

\section{$2.5{ }^{1} \mathrm{H}$ NMR 研究}

以 $\mathrm{CDCl}_{3} \mathrm{CD}_{3} \mathrm{OD}(V: V=7: 2)$ 作溶剂, 配制浓度 为 $1 \times 10^{-2} \mathrm{~mol} \cdot \mathrm{L}^{-1}$ 左右的主体分子钳溶液, 进行核磁 谱图的第一次测定. 测定完后, 加入与主体分子钳等物 质量的客体分子, 轻轻震荡, 使主客体充分络合. 放置 一段时间后，在同一条件下，进行磁谱图的第二次测定. 最后对比两次谱图的变化(主客体在配合前后的化学位 移变化). 我们通过主客体作用前后化学位移值的变化, 从而了解主体与客体分子发生作用的位置. 图 6 为分子 钳 $5 \mathrm{k}$ 与录离子配合前后的核磁对比图.

从表 5 中可看出, $\mathrm{Hg}^{2+}$ 对主体 $\mathbf{5 k}$ 的核磁的影响. 识 别作用前, 主体分子钳 $5 \mathbf{k}$ 中三唑环结构中的碳氢质子 $(\mathrm{CH})$ 的核磁化学位移值分别为 $\delta 8.076,7.856$. 配合后,
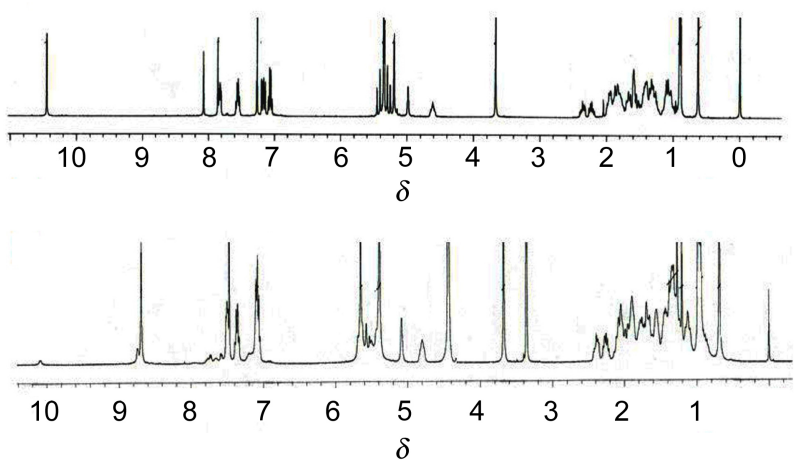

图 6 分子钳 $5 k(\mathrm{~A})$ 和 $5 \mathrm{k}$ 与采离子形成的配合物 $(\mathrm{B})$ 的核磁对 比图

Figure 6 Nuclear magnetic resonance spectra of $\mathbf{5 k}(\mathrm{A})$ and complex $\mathbf{5} \mathbf{k} \cdot \mathrm{Hg}^{2+}$ (B)

化学位移值分别为 $\delta 8.744,8.690$, 分别向低场移动 $\delta$ $0.6680,0.8340$, 分子钳 $\mathbf{5 k}$ 中的双醛羰基 $(\mathrm{CHO})$ 分别向 高场移动 $\delta 0.2000,0.2500$. 这充分说明主体 $\mathbf{5 k}$ 与客体 $\mathrm{Hg}^{2+}$ 之间发生了配合作用. 主体结构中的三唑环和醛羰 基共同与客体形成配位共价键，促使主客体之间达到了 很好的识别效果.

表 5 分子钳 $5 \mathbf{k}$ 与录离子形成配合物前后的部分核磁数据对 比图

Table 5 Nuclear magnetic resonance spectra data for the complex of host $\mathbf{5 k}$ with $\mathrm{Hg}^{2+}$

\begin{tabular}{lccc}
\hline \multicolumn{1}{c}{ Proton } & Host $\delta$ & Complex $\delta$ & $\Delta \delta_{\mathrm{H}}=\delta$ (complex) $-\delta$ (host) \\
\hline Triazole-H & 8.076 & 8.744 & 0.6680 \\
Triazole-H & 7.856 & 8.690 & 0.8340 \\
CHO & 10.45 & 10.25 & -0.2000 \\
CHO & 10.45 & 10.20 & -0.2500 \\
\hline
\end{tabular}

\section{3 结论}

利用点击化学, 设计合成了 12 个新的含有 $1,2,3$-三 唑结构的鹅去氧胆酸类分子钳人工受体. 通过紫外滴定 法以及核磁法我们考察了这种钳形人工受体对阳离子 的识别配合性能. 结果表明, 这类受体对金属阳离子具 有很好的识别能力. 特别是分子钳 $\mathbf{5 k}, \mathbf{5 1}$ 对 $\mathrm{Hg}^{2+}$ 有优良 的选择性识别能力, 最大结合常数可达 $8328.08 \mathrm{~L} \cdot \mathrm{mol}^{-1}$. 同时，具有不同的取代基的手臂的同类型分子钳受体对 金属离子的识别能力也是有差异的. 这种由于主体手臂 芳环取代基的位置变化所造成的微环境效应对识别性 能的影响, 不仅在理论上对研究人工受体的设计合成和 微环境效应具有重要意义, 而且使其在化学传感器领域 中具有潜在的应用前景.

\section{References}

[1] Clarkson. T. W. Am. J. Clin. Nutr. 1995, 61, 682.

[2] Tchounwou, P. B.; Ayensu, W. K.; Ninashvili, N.; Sutton, D.; En- 
viron. Toxicol. 2003, 18, 149

[3] An, L.; Cai, Y. H.; Yan, C. G. Chin. J. Appl. Chem. 2005, 22, 980 (in Chinese).

(安琳, 蔡亚华, 颜朝国, 应用化学, 2005, 22,980.)

[4] Silva, A. P. de; Gunaratne, H. Q. N.; Gunnlaugsson, T.; Huxley, A. J. M.; McCoy, C. P.; Rademacher, J. T.; Rice, T. E. Chem. Rev. 1997, 97, 1515 .

[5] Kolb, H. C.; Finn, M. G.; Sharpless, K. B. Angew. Chem., Int. Ed. 2001, 40, 2004.

[6] Demko, Z. P.; Sharpless, K. B. Angew. Chem., Int. Ed., 2002, 41, 2110.

[7] Lewis, W. G.; Green, L. G.; Grynszpan, F.; Radic, Z.; Carlier, P. R.; Taylor, P.; Finn, M. G.; Sharpless, K. B. Angew. Chem., Int. Ed. 2002, 41,1053 .

[8] Schweinfurth, D.; Hardcastle, K. L.; Bunz, U. H. F. Chem. Commun. 2008, 1053.

[9] Barreto, A. de F. S.; Vercillo, O. E.; Birkett, M. A.; Caulfield, J. C.; Wessjohann, L. A.; Andrade, C. K. Z. Org. Biomol. Chem. 2011, 9, 5024.

[10] Cecioni, S.; Faure, S.; Darbost, U.; Bonnamour, I.; Parrot-Lopez, H.; Roy, O.; Taillefumier, C.; Wimmerova, M.; Praly, J.-P.; Im- berty, A.; Vidal, S. Chem. Eur. J. 2011, 17, 2146.

[11] Maeda, C.; Yamaguchi, S.; Ikeda, C.; Shinokubo, H.; Osaka, A. Org. Lett. 2008, 10, 549.

[12] Wang, J.; He, X.; Gao, L.; Sheng, L.; Shi, X,; Li, J.; Chen, G. Chin. J. Chem. 2011, 29, 1227.

[13] Fazio, M. A.; Lee, O. P.; Schuster, D. I. Org. Lett. 2008, 10, 4979.

[14] Lowe, A. J.; Long, B. M.; Pfeffer, F. M. Chem. Commun. 2013, 49, 3376.

[15] Oh, H.; Han, S. K.; Kim. B. H. Heteroat. Chem. 2012, 23, 187

[16] Hu, J.; Lu, J. R.; Ju, Y. Chem. Asian J. 2011, 6, 2636.

[17] Verzele, D.; Madder, A. Eur. J. Org. Chem. 2013, 673.

[18] Jadhav, J. R.; Bae, C. H.; Kim. H. S. Tetrahedron Lett. 2011, 52, 1623.

[19] Li, W. N.; Xu, Q.; Li, Y.; Zhu, W.; Cui, J. C.; Ju, Y.; Li, G. T. Tetrahedron Lett. 2013, 54, 3868.

[20] Li, D. Z.; Yang, Y. X.; Yang, C.; Hu, B. W.; Huo, B. L.; Xue, L. W.; Wang, A. Z. Tetrahedron 2014, 70, 1223.

[21] Hu, J.; Zhang, M.; Yu, L. B.; Ju. Y. Bioorg. Med. Chem. Lett. 2010, 20,4342

[22] Kumar, A.; Pandey, P. S. Tetrahedron Lett. 2009, 50, 5842.

[23] Benesi, H. A.; Hildebrand, J. H. J. Am. Chem. Soc. 1949, 71, 2703.

(Qin, X.) 